\title{
Henri Lefebvre y el espacio social: aportes para analizar procesos de institucionalización de movimientos sociales en América Latina - La Organización Barrial Tupac Amaru (Jujuy-Argentina)
}

FERNANDA VALERIA TORRES*

\section{Resumen}

A través del presente trabajo, me propongo desarrollar parte de una propuesta teórica-analítica que permita operacionalizar la categoría espacio social en pos de contribuir al análisis de procesos de institucionalización al interior de los movimientos sociales. Específicamente, expondré una posible lectura de la obra "The production of space", de Henri Lefebvre (1991) que permita la aprehensión de su tríada conceptual: prácticas espaciales, representación del espacio y espacios de representación en torno a los conceptos de territorio y lugar para comprender la articulación entre espacio y procesos de institucionalización al interior de algunos movimientos sociales en América Latina. Para esto, dedicaré la primera parte del trabajo a la discusión teórica de la categoría espacio social de Lefebvre y su posible aplicabilidad en torno a los conceptos territorio y lugar. Luego, expondré la manera en la cual defino y comprendo la institucionalidad y su vinculación con comportamientos y recorridos posibles de ciertos movimientos sociales en América Latina. Por último, me dedicaré a delinear una propuesta de articulación que permita entender los procesos de construcción territorial de dichos movimientos sociales como vectores de procesos de institucionalización de sus prácticas y sentidos, a través del análisis de un caso: la Organización Barrial Tupac Amaru en la provincia de Jujuy.

Palabras clave: Movimientos sociales. Prácticas espaciales. Território. Representación del espacio.

\footnotetext{
* Universidad Nacional de La Plata/CONICET (Argentina)
} 
Henri Lefebvre and the social space:

contributions to the analysis of institutionalization processes of Latin America's social movements - Tupac Amaru neighborhood organization (Jujuy-Argentina)

\section{Abstract}

The purpose of this work is to develop part of a theoretical and analytical proposal that aimed at operationalizing the category 'social space' so that to contribute to the analysis of institutionalization processes among social movements. I will discuss, particularly, a possible reading of Henri Lefebvre's book "The production of space" (1991), one which allows to apprehend the conceptual triad: spatial practices, representations of space and spaces of representation in relation to the concepts of territory and place, in order to understand the connections between space and institutionalization processes within some social movements in Latin America. To this end, the first part of the work is devoted to a theoretical discussion of Lefebvre's category 'social space' and to its possible applicability to the concepts of territory and place. Then I will discuss the way as I define and understand institutionality and its connection with behaviors and possible trajectories of certain social movements in Latin America. Finally, I will outline a proposal for articulation that enables to understand the processes of territorial construction by such social movements as vectors of processes of institutionalization of their practices and meanings, through the analysis of a case: the neighborhood organization Tupac Amaru in the province of Jujuy.

Keywords: Social movements. Spatial practices. Territory. 


\section{Henri Lefebvre: espacio social. ¿Territorio y lugar?}

“(...) la práctica espacial consiste en una proyección sobre un campo (espacial) de todos los aspectos, elementos y momentos de la práctica social. En el proceso, estos se separaron el uno del otro, aunque esto no significa que el control general se abandonó ni por un momento (...)" (Lefebvre, 1991:8, traducción propia')

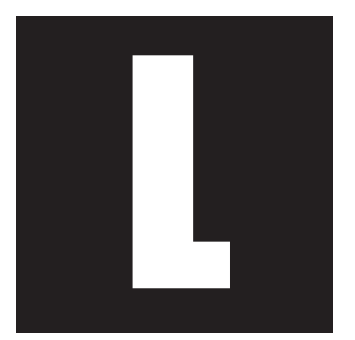

efebvre señala, en su búsqueda de una teoría única del espacio físico, mental y social, que esos espacios se pretenden, desde la teoría y desde la propia representación social, como espacios distinguibles y separados. Pero, desde la perspectiva del autor, debe defenderse una concepción del espacio como una integralidad multidimensional.

Parte, entonces, de la consideración del espacio social como un producto social y de allí, involucra los niveles analíticos necesarios para dar cuenta justamente del proceso de producción de dicho espacio social:

Si el espacio encarna las relaciones sociales, ¿cómo y por qué lo hace? ¿Y cuáles son esas relaciones? [...] Esto debe implicar la introducción de nuevas ideas - en primer lugar, la idea de una diversidad o multiplicidad de espacios bastante distinta de la multiplicidad que resulta de la segmentación y el seccionamiento del espacio ad infinitum [...] El espacio social se revela en su particularidad en la medida en que deja de ser indistinguible del espacio mental (según lo definen filósofos y matemáticos), por un lado, y del espacio físico (según la definición de la actividad práctico-sensorial

\footnotetext{
1 "Spatial practice consists in a projection onto a (spatial) field of all aspects, elements and moments of social practice. In the process these are separated from one another, though this does not mean that overall control is relinquished even for a moment: society as a whole continues in subjection to political practice -that is, to state power" (Lefebvre, 1991:8)
} 
y la percepción de la 'naturaleza'), por el otro. (Lefebvre, 1991:27, traducción propia)².

El concepto de práctica espacial nos permite concebir el espacio en tanto espacio social, es decir, como aquél en el cual se conjugan los diversos procesos y elementos de las relaciones sociales, aun cuando la relación de dominación pretenda presentarlos como elementos separados y autónomos. Las relaciones de poder tienen en el espacio un vehículo fundamental de naturalización del proceso de dominación que acompaña necesariamente al modo primordial de producción. El autor manifiesta con suma claridad cómo se relaciona el ejercicio de la hegemonía con el espacio:

¿Es concebible que el ejercicio de la hegemonía podría dejar el espacio sin tocar? ¿Podría el espacio no ser más que el locus pasivo de las relaciones sociales, el medio en el que su combinación adquiere lugar, o la suma de los procedimientos empleados en su eliminación? La respuesta es no. Más adelante voy a demostrar el activo rol - operativo o instrumental - del espacio, como conocimiento y como acción, en el modo de producción existente. Voy a mostrar cómo sirve el espacio y cómo la hegemonía hace uso del mismo, en el establecimiento, sobre la base de una lógica subyacente y con la ayuda de los conocimientos y la experiencia técnica, de un 'sistema' (Lefebvre, 1991:11, traducción propia) ${ }^{3}$

\footnotetext{
2 "If space embodies social relationships, how and why does it do so? And what relationships are they? (...) This must involve the introduction of new ideas -in the first place the idea of a diversity or multiplicity of spaces quite distinct from that multiplicity which result from segmenting and cross-sectioning space and infinitum (...) social space will be revealed in its particularity to the extent that it cease to be indistinguishable from mental space (as defined by the philosophers and mathematicians) on the one hand, and physical space (as defined by practico-sensory activity and the perception of "nature") on the other." (Lefebvre, 1991:27)

3 "Is it conceivable that the exercise of hegemony might leave space untouched? Could space be nothing more than the passive locus or social relations, the milieu in which their combination takes on body, or the aggregate of the procedures employed in their removal? The answer must be no. Later on I shall demonstrate the active -the operational or instrumental -role of space, as knowledge and action, in the existing mode of production. I shall show how space serves and how hegemony makes use of it, in the establishment, on the basis of an underlying logic and with the help of knowledge and technical expertise, of a 'system'"( Lefebvre, 1991:11).
} 
Luego el autor aclara que este uso hegemónico del espacio no implica que se esté frente a un espacio libre de contradicciones, por el contrario, el espacio es un terreno de disputas constantes por su definición, su uso y su control.

Toda sociedad produce su propio espacio. Más específicamente podemos decir que cualquier modo de producción, junto con sus específicas relaciones de producción, necesitan de un espacio. Lefebvre incluso postula que es a través del espacio como se distinguen dos relaciones fundamentales en la sociedad, asignándoles un lugar determinado, localizándolas en forma separada y, en dicha separación, definiéndolas: las relaciones de reproducción y las relaciones de producción. Esta situación se complejiza aún más con el advenimiento del capitalismo, cuando deben distinguirse tres relaciones fundamentales: la reproducción biológica (la familia); la reproducción de la fuerza laboral (fundamentalmente la clase trabajadora) y la reproducción de las relaciones sociales de producción.

En este punto, para comprender el mencionado proceso de producción del espacio, Lefebvre introduce una "tríada conceptual" que será estructurante de toda su obra:

1- Prácticas espaciales (el espacio percibido): abarca la producción y la reproducción, los lugares concretos y las características de los conjuntos espaciales de cada formación social. Aseguran la continuidad y cierto grado de cohesión. En términos de espacio social, y de cada miembro de la relación de una sociedad dada a ese espacio, esta cohesión implica un nivel garantizado de competencia y un nivel específico de rendimiento.

2- Representaciones del espacio (el espacio concebido): están vinculadas a las relaciones de producción y al "orden" que imponen esas relaciones y, por lo tanto, al conocimiento, a los signos, a los códigos y a las relaciones «frontales». "Espacio conceptualizado, el espacio de los científicos, de los planificadores, urbanistas, técnicos e ingenieros sociales 
(...) quienes identifican lo que es vivido y percibido con lo que es concebido" (Lefebvre, 1991:38). Se trata del espacio dominante en cualquier sociedad (o modo de producción) y es fundamental su influencia en el proceso de producción del espacio y en la actividad productiva de la sociedad.

3- Espacios de representación (el espacio vivido): incorporan simbolismos complejos, a veces codificados, a veces no, vinculados al costado clandestino o marginal de la vida social, como también al arte. Los espacios de representación producen, generalmente, resultados simbólicos.

El espacio social, que es la conjugación de todas estas dimensiones, es una herramienta para el análisis de la sociedad. Las prácticas espaciales, representaciones del espacio y espacios de representación contribuyen en diferentes formas a la producción del espacio de acuerdo a sus cualidades y atributos, de acuerdo a la sociedad o el modo de producción en cuestión y de acuerdo al período histórico. De esta manera el autor caracteriza el espacio construido en la sociedad de la edad media, en la sociedad capitalista y en una posible sociedad socialista.

Una revolución que no produzca un nuevo espacio no podrá realizarse en todo su potencial; de hecho, fracasaría al no poder cambiar la vida misma, sino meramente superestructuras ideológicas, instituciones o aparatos políticos.

Es clara la centralidad del espacio para desarrollar procesos de cambio estructurales, y la necesidad de identificar la lucha de clases en y por el espacio, por su definición, por sus usos, es decir, la lucha de clases que interviene en la producción del espacio, como aquella que puede vencer al espacio indiferenciado construido por el modo de producción capitalista, al espacio dominante en dicha sociedad: el espacio abstracto:

Hoy, más que nunca, la lucha de clases se inscribe en el espacio. De hecho, es esta lucha la que impide que el 
espacio abstracto se apodere de todo el planeta y tape todas las diferencias. Sólo la lucha de clases tiene la capacidad de diferenciar, de generar diferencias que no son intrínsecas al crecimiento económico qua estrategia, "lógica" o "sistema" -es decir, diferencias que no son inducidas por, ni aceptables para dicho crecimiento. Las formas de la lucha de clases son ahora mucho más variadas que antes. Naturalmente, incluyen la acción política de las minorías. (Lefebvre, 1991:55, traducción propia) ${ }^{4}$

Nos proponemos traducir la propuesta de Lefebvre en torno al espacio social en dos conceptos de un nivel de generalidad menor que son, por supuesto, distinguibles analíticamente pero que conviven entramados y superpuestos en la vida real: territorio y lugar, siendo nuestro particular interés en este trabajo resaltar la importancia del primero para poder comprender las acciones políticas.

La especificidad del territorio proviene de su asociación con las relaciones de poder, es decir, permite introducir la variable política al pensar el espacio construido en tanto territorio como producto de relaciones de poder, de dominación y resistencia (Raffestin, 1993; Sack, 1986; Santos, 2000, entre otros). Bajo esta definición, puede haber varios territorios en un mismo espacio, porque para que haya territorio, el límite debe ser usado para controlar su acceso; en términos generales podemos decir que tiene que existir una relación de poder, una relación de dominación actuando tras la delimitación y ejercicio de un territorio.

Lo que distingue al concepto de lugar, por otra parte, es el proceso de identificación con un determinado espacio a través de diversos instru-

\footnotetext{
4"Today more than ever, the class struggle is inscribed in space. Indeed, it is that struggle alone which prevents abstract space from taking over the whole planet and papering over all differences. only the class struggle has the capacity to differentiate, to generate differences which are not intrinsic to economic growth qua strategy, 'logic' or 'system' -that is to say, differences which are neither induced by nor acceptable to that growth. The forms of the class struggle are now far more varied than formerly. Naturally, they include the political action of minorities" (Lefebvre, 1991:55).
} 
mentos simbólicos y experienciales: nos referimos a los sentimientos de pertenencia o extrañamiento, de apego o rechazo frente a un espacio definido de acuerdo a la carga de experiencias, emociones, representaciones que ofrecen la posibilidad de una relación identitaria, o no, con el mismo.

Lefebvre distingue dos formulaciones que pueden abonar este mismo sentido:

- los espacios apropiados, utilizados para servir las necesidades y posibilidades de una comunidad, espacios que posibilitarían una "apropiación" simbólica y de identidad además de funcional, apropiación que sólo puede tener inicio en el "lugar", en tanto lo local, lo cotidiano de cada individuo, $y$

- los espacios dominados, espacios transformados y dominados que son habitualmente cerrados, utilitarios y funcionales: pensados para controlar procesos naturales y sociales para la producción.

Podemos afirmar que el concepto de territorio supone un espacio determinado y controlado socialmente, supone la construcción de un espacio en el cual se ejerzan relaciones de poder que permitan su control, la definición de quienes tienen acceso a él y quienes no, la determinación de sus usos posibles. El territorio, entonces, puede entenderse como dimensión constitutiva de la dominación y, en última instancia, del atributo de lo político; se involucra en el ejercicio y la reproducción de la dominación. El espacio territorializado tiene una capacidad operativa peculiar para abonar la constitución del modo de producción en tanto sistema de dominación, al operacionalizar espacialmente las relaciones de poder. Si nos referimos y analizamos las instituciones políticas imperantes en una sociedad, entendemos que debemos referirnos y analizar la producción de un espacio territorial que involucra la posibilidad de dichas instituciones. 
Instituciones e institucionalización de movimientos sociales en América Latina

El particularismo o clientelismo que señala O'Donnell, en su trabajo ya clásico denominado "Otra institucionalización", coexiste en incómoda tensión con y dentro de las instituciones formales de lo que el autor denomina el complejo institucional de la poliarquía, es decir, de la democracia. Por eso habla de las democracias "institucionalizadas informalmente" (O'Donnell, 1997: 307), aludiendo a ciertas prácticas fuertemente arraigadas en las prácticas políticas de varios de los países de Latinoamérica, que pueden entenderse como instituciones no formales (aludiendo con el término formal al complejo institucional poliárquico).

Esta misma idea es la que propongo retomar para analizar las formas institucionales que adoptan los movimientos sociales, sobre todo, en contextos en los cuales el Estado es excedido por demandas, expectativas e interrogantes a los que no puede hacer frente de manera exitosa.

Apartándome de las orientaciones paradigmáticas para analizar los movimientos sociales, el enfoque del proceso político y las teorías de los nuevos movimientos sociales, en este trabajo postulo analizar la emergencia, en Argentina, de diversos movimientos sociales, teniendo en cuenta el contexto de profunda crisis social, económica y cultural que supusieron las grandes transformaciones neoliberales operadas en nuestro país, pero sin acordar con que dicha emergencia supone una alternativa representativa frente a la crisis de actores institucionales considerados clásicos en esa temática...sindicatos y partidos.

Claus Offe es un claro exponente de esta idea, centrado en el caso europeo: El campo de la acción de los nuevos movimientos es un espacio de política no institucional, cuya existencia no está prevista en las doctrinas ni en la práctica de la democracia liberal y del Estado de Bienestar (Offe, 1992:174). 
Charles Tilly, ubicado en la tradición norteamericana de análisis de la acción social, expresa en las palabras finales de su libro que [...] la profesionalización conduce a la institucionalización, y, por lo tanto, al descenso en términos de innovación en los movimientos sociales (Tilly, 2010: 299), reafirmando la ligazón entre institucionalización y rutinización, burocratización y previsibilidad, con la consiguiente pérdida de la capacidad innovadora que caracterizaría a los movimientos sociales. En la misma línea, Tarrow argumenta: [...] los movimientos institucionalizan sus tácticas e intentan obtener beneficios concretos para sus seguidores a través de la negociación y el compromiso. Se trata de un camino que a menudo conduce al éxito a costa de transformar el movimiento en un partido o un grupo de interés (Tarrow, 1997: 200)

Podríamos decir que tanto los exponentes del Ilamado Enfoque del Proceso Político, como los autores de la teoría de los Nuevos Movimientos Sociales, han brindado poca atención al análisis de las interacciones entre los Movimientos Sociales y las instituciones políticas, partiendo del supuesto de separación entre Sociedad civil y Estado. Los primeros, al enfatizar la evaluación de la acción colectiva como mecanismo de conflicto político contra los detentores del poder (McAdam et al, 2005; Tarrow, 1997), y los segundos, al resaltar las discontinuidades de las nuevas expresiones movimientistas respecto a las modalidades tradicionales de acción política (Touraine, 1985; Offe, 1992), dificultan el reconocimiento de las relaciones de reciprocidad de diverso sentido que protagonizan los movimientos sociales y el Estado.

Por el contrario, asumimos que los movimientos sociales son aquellos sectores organizados de la sociedad que emergen dando cuenta de nuevas expectativas, interviniendo y reformulando el espacio de mediaciones clásicas entre la sociedad civil y el estado, distinguiéndose de las ideas más tradicionales en torno a la figura del ciudadano y de los trabajadores, conjugando 
de diversos y divergentes modos el lazo de representación social y, en ocasiones, político que, las más de las veces no presentan una alternativa (en el sentido de esto o lo otro) sino que comprenden solapamientos y superposiciones con las formas consideradas más tradicionales de representación.

Considero que debe ser revisado el concepto de política no institucional para pensar las formas de acción política en América Latina, región en la cual el funcionamiento real de la democracia liberal supuso, al menos, corrimientos, respecto al "modelo" europeo, para muchos "espejo" frente al cual mirarse e imitar.

Es, en este sentido, saludable el aporte de varios autores brasileros (Silva; Oliveira, 2011; Carlos, 2015; 2011; Abers; Von Bülow, 2011; Dowbor, 2014) preocupados por aportar a la comprensión de la relación entre el Estado y los movimientos sociales y sus dinámicas de institucionalización. Silva y Oliveira (2011) dan cuenta de aquellos escenarios en los que se desarrolla un complejo entramado entre el Estado, los partidos políticos y los movimientos sociales, que proponen denominar como patrón de relación marcado por la interpenetración, en el cual la frontera entre partidos y movimientos se torna difusa por la intersección de esferas de acción y una militancia "múltiple". Dicha militancia y específicamente el rol desempeñado por el Partido dos Trabalhadores (PT), en el caso del Movimiento de Economía Solidaria de Rio Grande do Sul, posibilita un fluido "tránsito institucional" de ciertos actores y militantes, compartidos por el partido y el movimiento y que resulta en una estrategia exitosa por parte de los movimientos para alcanzar sus objetivos. Con igual sentido crítico ante las posturas teóricas que suponen una separación entre los movimientos sociales y la política institucional, Carlos $(2011$; 2015) propone la comprensión de su contingencia y mutua constitución, reconociendo patrones de acción colectiva complejos y contradictorios en lo atinente a la relación sociedad-Estado. La autora resalta una visión compleja 
del proceso de inserción institucional de los movimientos, el cual no sólo incide en la dimensión organizacional, sino también en las dimensiones relacional y discursiva de la acción colectiva. Y plantea que la inserción institucional puede provocar cambios en los patrones de acción colectiva, llevando a modelos híbridos en los cuales se combina la contestación y la cooperación, la autonomía y la influencia en la agenda pública. Por su parte, Abers y Von Bülow (2011) dan cuenta de la importancia del análisis de las redes de acción colectiva que existen en la práctica y que permiten comprender a los activistas dentro del Estado, es decir, que participan directamente de la gestión estatal como parte de la misma unidad de análisis al estudiar los movimientos sociales como "redes de actores"; discutiendo con las tesis que presumen una relación siempre conflictiva entre los movimientos sociales y el Estado y con las tesis que resaltan la cooptación o colonización de la lógica jerárquica del estado sobre los movimientos. Por último, Monika Dowbor (2014) analiza la trayectoria del movimiento sanitario brasileño en las tres últimas décadas y propone la incorporación de las prácticas institucionalizadas de acción y las acciones que ocurren vía instituciones al concepto de repertorio de acción colectiva. Su trabajo muestra que, en el contexto democrático, una de las modalidades de acción institucionalizada del movimiento sanitario es la ocupación de cargos en el Estado; una táctica utilizada por el colectivo para el éxito de su proyecto político y que también es utilizada por otros movimientos.

Considerando las acciones desarrolladas por algunos movimientos sociales en América Latina, podríamos decir que no solo corrieron el eje de ciertas demandas culturales, ideológicas y simbólicas, sino que muchos de dichos movimientos lograron operar transformaciones concretas en los vínculos entre el Estado, el espacio público y la institucionalización de prácticas colectivas. 
Volviendo al concepto de institución y a O'Donnell, el autor nos brinda una definición:

Por institución, entiendo una pauta regularizada de interacción conocida, practicada y aceptada (si bien no necesariamente aprobada) por actores cuya expectativa es seguir actuando de acuerdo con las reglas sancionadas y sostenidas por ella. [...] Las instituciones "están allí", regulando expectativas y comportamientos que no cuestionan su existencia socialmente determinada (O'Donnell, 1997: 310).

Cuando las reglas informales están generalizadas, son ampliamente compartidas y determinan las expectativas de acción, estas son las que deben considerarse altamente institucionalizadas, no las formales. Por eso, analizando el recorrido protagonizado por ciertos movimientos sociales en Argentina en la última década, podemos identificar ciertas prácticas y sentidos que han ido cobrando un profundo carácter institucional (informal): la movilización social y los procesos de territorialización protagonizados por parte de algunos movimientos sociales se cuentan entre ellos, de acuerdo a nuestro análisis. De estos dos procesos, nos ocuparemos solo del segundo en el siguiente apartado, pero antes deberemos especificar el enfoque propuesto.

La primera aclaración necesaria es nuestra posición crítica respecto a asociar el análisis de procesos de institucionalización de movimientos sociales como cooptación de una fracción o partido político, o como sumisión/integración al gobierno. Por el contrario, tal como nos enseña O’Donnell, las instituciones deben considerarse como una mediación entre la sociedad civil y el régimen político, de tal modo que puedan ser redefinidas las formas de participación y los dispositivos de legitimación que conforman una comunidad política (O’Donnell, 1993; 1997). El interrogante por la institucionalización supone atender a los efectos de un ciclo de movilización sobre el funcionamiento de ese régimen político. 
Respecto a la caracterización del ciclo de movilización abierto en Argentina hacia finales de la década del noventa y que puede darse como un segundo momento de clausura promediando el gobierno kirchnerista, podemos señalar que el mismo representa la apertura hacia el espacio público de voces hasta ese momento marginadas: sectores recientemente desocupados, trabajadores precarizados, colectivos identitarios marginados (étnicos, culturales, de género, etc.) y podríamos seguir enumerando otros. Dicho ciclo de movilización, junto con la transformación de los tipos de protesta ejercidos, las demandas motorizadas y, como acabamos de nombrar, los sujetos protagonistas, supuso el ejercicio de la influencia sobre los temas de agenda pública que debían comenzar a ser atendidos. Así, las demandas por trabajo digno, por espacios culturales legítimos, por el amparo de actividades audiovisuales alternativas, por el reconocimiento social y legal a la diversidad sexual, a la diversidad étnica, entre otros, comenzaron a formar parte, con un lugar protagónico, de los debates sociales y políticos en diversos ámbitos de la sociedad argentina.

Nos encontramos hablando de ciclos de movilización social que suponen la apertura de espacios para la intervención política mediante mecanismos y herramientas que exceden, por mucho, la participación electoral, factor determinante, desde el punto de vista de la acción de los ciudadanos, en los enfoques poliárquicos sobre la democracia. Luis Tapia postula que, en las sociedades modernas, se eligió al Estado como el lugar privilegiado para la política; sin embargo, las complejidades que fueron transformando a la sociedad han ampliado esos lugares predilectos para la política, sobre todo de la mano de los procesos de ciudadanización, de los procesos de ampliación de la condición y el contenido del rol de la ciudadanía. En esta dirección, la sociedad civil es, tal como expresa el autor, "otro lugar de la política"; es decir, es un espacio no estatal, que se rige con otras pautas, pero un espacio para la política al fin. 
Al ampliar la perspectiva, la institucionalización no queda reducida al espacio de lo estatal, sino que es posible pensarla como estabilización o como establecimiento de ciertas regularidades, pautas comunes en escenarios propios de la sociedad civil, que se hallan politizados. Tapia agrega que los movimientos sociales no tienen un lugar específico donde hacer política, por ello pueden transitar la sociedad civil e incluso el estado politizando los espacios sociales "con sus críticas, demandas, discursos, prácticas y proyectos" (Tapia, 2008:56).

Podemos analizar el comportamiento de algunos movimientos sociales que entienden a la sociedad civil como otro espacio legítimo de la política a través del cual poder regularizar y establecer pautas comunes de acción no estatales, trascendiendo no sólo el estadocentrismo de la política, sino también el estatuto destituyente e instituyente, bajo la forma de una nueva propuesta de institucionalización.

La constitución de los movimientos sociales es un desplazamiento de la política, de los lugares institucionalizados de la misma, al campo de tránsito entre ellos y al de la fluidez. También es un modo de politización de lugares sociales o conjunto de estructuras y relaciones sociales que habían sido neutralizadas o despolitizadas y, por tanto, legitimadas en su forma de organización de algunas desigualdades (Tapia, 2008:56)

Esta perspectiva de pensamiento nos habilita a realizar el último giro conceptual para hilar el concepto territorio y su fuerte ligazón con las relaciones de poder y el atributo de lo político, con el análisis de los mencionados procesos de institucionalización informales desarrollados por algunos movimientos sociales en nuestro país durante los últimos años. 


\section{Movimientos sociales: territorios e instituciones. Vinculaciones y tensiones}

Retomando lo ya expresado en torno al enfoque analítico que nos propone Lefebvre para definir el espacio social como una categoría fundamental para comprender el entramado de relaciones y disputas que supone la vida social, podremos ahora focalizarnos en el territorio. Se establece su delimitación a partir de relaciones de conflicto y confrontación, las cuales definen los sujetos que dominan el territorio y controlan el acceso al mismo. Podemos identificar en un mismo espacio diversas clases y relaciones sociales que producen diferentes espacios y territorios, y que se reproducen en permanente conflictualidad.

Nos dice Fernandes: A disputa territorial ocorre de dois modos: pela desterritorialização ou pelo controle das formas de uso e de acesso aos territórios; ou seja, controlando as suas territorialidades ${ }^{5}$ (Fernandes, 2009:208). Pueden encontrarse diversos estudios realizados por geógrafos y sociólogos que examinan esas disputas y conflictualidades. Indígenas, campesinos y movimientos urbanos sin techo, en el bosque, en el campo y en la ciudad, disputando territorios a fin de garantizar su existencia a partir de sus identidades. Puede observarse aquí, desde otro ángulo, como opera la condición ya señalada de la integralidad del espacio social: una integralidad y multidimensionalidad que supone su entendimiento en tanto lugar, y de allí se construyen identidades colectivas, y en tanto territorio, y de allí la necesidad de imprimir sobre él una relación de poder en pos de su defensa o reproducción.

\footnotetext{
5 "La disputa territorial se produce de dos maneras: por la desterritorialización o por el control de las formas de uso y de acceso a los territorios, o sea, controlando a sus territorialidades." Versión en castellano traducida por el mismo autor, disponible en http://web.ua.es/es/giecryal/ documentos/documentos839/docs/bernardo-tipologia-de-territorios-espanol.pdf
} 
La expansión y / o creación de territorios son acciones en las que se explicita la conflictualidad y las contradicciones de las relaciones socioespaciales y socioterritoriales. Estas relaciones permiten distinguir, a su vez, los movimientos socioespaciales de los movimientos socioterritoriales. Algunos movimientos sociales transforman el espacio en territorio a través de la conflictualidad entre las fuerzas políticas que intentan crear, conquistar y controlar sus territorios. Los movimientos sociales también forman parte de los procesos geográficos de T-D-R: se territorializan y son desterritorializados y se reterritorializan. Todos los movimientos, de acuerdo a esta perspectiva, son socioespaciales, porque producen espacios, pero no todos los movimientos son socioterritoriales. Los movimientos socioterritoriales, para alcanzar sus objetivos políticos, construyen espacios políticos, se espacializan y promueven otro tipo de territorio (procesos de Territorialización-Desterritorialización). Tienen el territorio no solamente como objeto, sino que éste es esencial para su existencia: luchan para conquistar el territorio o, como está expresado en el título de éste apartado, para estos movimientos "el territorio es la vida".

Los procesos de territorialización - desterritorialización - reterritorialización (T-D-R), que se constituyen a partir de la expansión y la destrucción; la creación y el reflujo que describen entre otros Fernandes (2005; 2009); Haesbaert (2004); Hiernaux y Lindón (2004) ayudan a echar luz sobre lo que aquí se quiere identificar.

Nos referimos a los espacios sociales que fueron abandonados por el Estado a lo largo de décadas y que, no obstante la transformación innegable que supone la parcial recuperación del Estado como herramienta de acción e intervención durante los últimos gobiernos nacionales de Néstor Kirchner y Cristina Fernández de Kirchner, aún en la actualidad permanecen espacios en los que no se halla sino ausencia y desidia estatal, espacios desterritorializados desde el punto de vista de las relaciones políticas que operan en su definición y en el anclaje posible de los sujetos que lo habitan, lo producen y lo viven. 
Este es el "escenario" en el cual operan los movimientos sociales y sus prácticas espaciales de reterritorialización, es decir, de nuevas vinculaciones con el territorio que permiten nuevas definiciones de sus límites, su acceso, formas y posibilidades de su uso y su control. Consideramos, entonces, que uno de los dispositivos de institucionalización no formal de los movimientos sociales, entendiéndolos como sujetos políticos, es el que se desarrolla a través de los procesos de reterritorialización. Un movimiento social exige un reordenamiento pequeño o grande de la sociedad y del estado, y empieza haciéndolo a través del desbaratamiento de las relaciones políticas de poder establecidas para la reproducción de las desigualdades existentes (Tapia, 2008: 60). En el desbaratamiento de las relaciones políticas de poder territoriales que, por supuesto, implican relaciones de desigualdad y dominación, ubicamos el caso que nos ocupa.

Proponemos pensar el proceso de desterritorialización protagonizado por el Estado como la experiencia que, durante la década neoliberal, marcó una forma de llevar adelante una nueva configuración de la dominación social: fortaleciendo a sectores económicos poderosos y globales, quienes llevaron adelante un fuerte proceso de reterritorialización, a través de formas de intervención económica con un sesgo marcadamente extractivista y desindustrializante, y abandonando en términos de gestión y de recursos a todas las expresiones económicas consideradas improductivas o poco rentables, ineficientes y dependientes. Esta nueva configuración territorial traerá consigo una nueva configuración de la desigualdad, donde la escala local y regional se verá especialmente afectada por la "retirada tendenciosa" ${ }^{6}$ del Estado.

\footnotetext{
${ }^{6}$ Retirada tendenciosa, porque el Estado no siempre dejará el espacio "vacante", sino que en muchas ocasiones su corrimiento es originado por el facilitamiento de incorporación de otros agentes de poder e influencia. Uno de los ejemplos más claros está dado por los procesos de privatizaciones de empresas estatales, las cuales se realizan con compradores previamente acordados y bajo condiciones absolutamente favorables a sus intereses. Otro ejemplo paradigmático es la adecuación de la legislación de tierras para la implantación de empresas extractivas, en el rubro de la minería y los hidrocarburos fundamentalmente (ver Svampa, 2012 y Machado Aráoz, 2012).
} 
Dicha experiencia será la plataforma de constitución de procesos de reterritorialización por parte de movimientos sociales, como la Organización Barrial Tupac Amaru (OBTA) en Argentina, que suponen claramente la emergencia de, citando nuevamente a $\mathrm{O}^{\prime}$ Donnell, una pauta regularizada de interacción conocida, practicada y aceptada (si bien no necesariamente aprobada) por actores cuya expectativa es seguir actuando de acuerdo con las reglas sancionadas y sostenidas por ella. Es decir, la emergencia de otras instituciones para atender la resolución de diversos problemas y necesidades sociales: trabajo, vivienda, salud, educación. Y dicha institucionalización informal será vectorizada, a partir de procesos de reterritorialización protagonizados por la organización.

\section{El proceso de territorialización protagonizado por la OBTA}

La OBTA es una organización que se autodefine como "barrial" y nace en la provincia de Jujuy ${ }^{7}$, provincia ubicada en el extremo norte de Argentina, al calor de las luchas protagonizadas por diversos colectivos y sujetos sociales golpeados por las políticas neoliberales llevadas adelante con inusitada profundidad en nuestro país durante la década de los noventa. La provincia de Jujuy representa una de las regiones más pobres del país, se trata de una provincia con casi nulo desarrollo industrial, dependiente en gran medida del empleo estatal y que, ante el achicamiento y la retirada del Estado llevadas adelante durante la implementación del ordenamiento neoliberal, se vio seriamente afectada en términos de desocupación, niveles de pobreza y calidad de los servicios públicos (Lagos, 2009; Bergesio; Golovanevsky, 2014)

\footnotetext{
${ }^{7}$ La provincia de Jujuy se encuentra ubicada en el noroeste de la República Argentina, tiene una superficie de $53.219 \mathrm{Km} 2$ y una población cercana a los 611.888 habitantes. Su ciudad capital es San Salvador de Jujuy, fundada en 1593.
} 
La Tupac Amaru surgió a fines de 1999, acompañando las demandas de mercadería y obtención de Planes Sociales de atención al desempleo ${ }^{8}$, durante el gobierno nacional de Fernando De la Rúa (1999-2001) y el posterior de Eduardo Duhalde (2002-2003). Su principal referente es Milagro Sala, hasta ese momento militante de un sindicato de empleados estatales (Asociación Trabajadores del Estado, ATE), protagonista de las luchas provinciales enfrentando el ajuste neoliberal. Ella promueve la creación de la OBTA a los fines de organizar los barrios de Jujuy. Milagro, "la Mila" o "la flaca" como se la conoce, posee una capacidad de liderazgo indiscutido; liderazgo carismático y personalista, asociando afectividad, capacidad inagotable de trabajo, disciplina y rigidez.

En el 2004, la organización se adaptó al modelo cooperativo para poder cumplir con el Programa Federal de Emergencia Habitacional para la construcción de viviendas, programa desarrollado por el entonces presidente Néstor Kirchner. La Tupac Amaru se asume como una organización fuertemente comprometida con el proyecto nacional que se pregona desde el gobierno nacional de Néstor Kirchner y que continuará de igual forma durante los gobiernos posteriores de Cristina Fernández de Kirchner. Esta situación traerá aparejada una suerte de "tensión escalar" en su relación con el Estado ${ }^{9}$, puesto que a nivel nacional es una organización que se ubica en una clara alineación y apoyo, pero que confrontará fuertemente con el mismo partido a nivel provincial y municipal (bajo los gobiernos justicialistas provinciales de Eduardo Fellner (2003-2007)

\footnotetext{
${ }^{8}$ Planes estrenados por el gobierno de Carlos Menem durante su segundo mandato (19951999), en el marco del programa que se denominó inicialmente Programa Trabajar. Estos seguirán vigentes durante el gobierno de De la Rúa con la incorporación de otros programas a nivel nacional y provinciales: un trabajo publicado en el 2002 señala: "Actualmente existen 28 programas de empleo provinciales, y desde 1993 hasta la fecha se crearon más de 20 programas nacionales" (Ronconi, 2002: 4)

${ }^{9}$ Es interesante el análisis que sobre este fenómeno realiza Lorena Moscovich (2009).
} 
y Walter Barrionuevo (2007-2011) y el gobierno municipal de José Luis Martiarena (2003-2007).

Lo que aquí interesa resaltar es el nivel de desarrollo alcanzado por la organización en la provincia, que puede resumirse a partir de un dato revelador: la OBTA es el tercer empleador a nivel provincial (luego del Estado y la Empresa Ledesma), tiene alrededor de 70.000 afiliados en Jujuy y 150.000 si se cuentan las otras provincias (OBTA, 2014:2). Pero no sólo es importante atender la dimensión cuantitativa: la organización ha establecido un sistema de gestión y resolución de los principales problemas que aquejan a los sectores populares en una buena parte del territorio de la provincia, a los que el Estado ha dejado sin respuesta desde hace décadas (y cuya desidia fue profundizada a partir de los años noventa). Nos referimos a problemas en torno, como ya dijimos, al empleo; problemas en torno a la atención de la salud; al acceso a la educación y al acceso a la vivienda propia. La resolución de esta última problemática, como analizaré a continuación, se desarrollará como un eslabón fundamental para comprender el proceso (y el poder) territorial construido por la OBTA.

La Tupac Amaru comienza a construir viviendas en el año 2004, a partir de luchar por la obtención directa del financiamiento que provenía del Ministerio de Planificación Federal, Inversión pública y Servicios de la nación, con intervención del Ministerio de Desarrollo Social, a través de la creación de cooperativas. Luego de corroborar que la organización logró construir las viviendas acordadas no sólo en un plazo menor al pautado sino con un costo también menor, el financiamiento se fue incrementando a lo largo de los años para la realización de más y más viviendas que fueron abriendo la posibilidad de crecimiento de la Tupac Amaru.

A través de la construcción de viviendas a un costo menor, la organización pudo contar con dinero de saldo con el cual construir ya no casas sino escuelas, centros de salud, polideportivos, fábricas, etc. Así lo resume 
uno de los referentes principales de la organización: "La clave nuestra está en la posibilidad de financiarnos a través del ahorro que ganamos con cada vivienda que hacemos a un costo más bajo. Y ese ahorro lo pusimos en infraestructura y servicios para la gente" (Ricardo, S. S. de Jujuy, 09/04/15 ${ }^{10}$ ). Han construido 8000 viviendas en toda la provincia, a través de, en la actualidad un total de 157 cooperativas (cinco cooperativas textiles y el resto cooperativas de vivienda) (OBTA, 2014:4).

Si bien todo este proceso despierta muchos interrogantes a ser atendidos, aquí analizo del proceso de territorialización que supuso la construcción de estos barrios de vivienda de la Tupac, junto con el desenvolvimiento de las copas de leche de la organización ${ }^{11}$. Específicamente nos detendremos en el caso del Barrio Alto Comedero de San Salvador de Jujuy, por representar el ejemplo que cuantitativa y cualitativamente tiene mayor trascendencia.

La ciudad de San Salvador de Jujuy, ciudad capital de la provincia, con 231.229 habitantes $^{12}$, representa el $37.8 \%$ de la población de Jujuy, formando junto con las vecinas localidades de Palpalá y Yala un aglomerado (Gran San Salvador de Jujuy) de 278.336 habitantes (45.5\% de la población provincial). Supone un centro de atracción de población por las esperanzas cifradas en torno a la obtención de empleo, posibilidades educativas,

\footnotetext{
${ }^{10}$ Los nombres reales de las personas entrevistadas han sido modificados para preservar su identidad.

${ }^{11}$ Las copas de leche funcionan como una suerte de núcleo básico de la organización, se trata de centros barriales en los cuales se brinda la leche y en ocasiones también el almuerzo a los vecinos pobres del barrio. Fueron el origen de la organización y, si bien luego conformaron las cooperativas de trabajo estas copas de leche se mantienen como el bastión territorial fundamental. Todos los afiliados a la organización, lo hacen a través de una copa de leche, aún en los casos en los que no realizan un trabajo concreto en ella. Cooperativistas que forman parte de una cuadrilla de construcción, realizan su jornada laboral en dicha cuadrilla, pero pertenecen y tienen que cada tanto colaborar con la copa de leche a la que pertenecen.

${ }^{12}$ Censo Nacional de Población, Hogares y Vivienda 2001 (INDEC)
} 
obtención de recursos estatales que no llegan a los diversos rincones de la provincia. Sin embargo, y tal como se repite en casi todas las ciudades intermedias de nuestro país ${ }^{13}$, la posibilidad de obtener una vivienda digna es cada vez una expectativa con mayores dificultades de realización.

Sobre este escenario, la OBTA, se lanzó a la obtención de tierra urbana disponible para construir las primeras viviendas que fueron financiadas. Todos los relatos se asemejan:

Hasta que se dio: 'vayan a buscar terrenos'. Andábamos por todos lados, buscando terrenos que sean terrenos fiscales, que no tengan dueño o si tenía dueño teníamos que preguntar si tenían las 7 hectáreas, me acuerdo que buscábamos las 7 hectáreas por todos lados... Un grupito salía para un lado, otro grupito salía para otro lado buscando terrenos. Hasta que un día se dio que encontramos las tierras de Alto Comedero, nos mandaron a desmalezar y dijimos bueno acá vamos a hacer las viviendas (María, Área de administración de las cooperativas, S. S. de Jujuy, 13/04/2015).

Finalmente, ocuparon un predio ubicado en el barrio Alto Comede$\mathrm{ro}^{14}$, en la parte "alta" de San Salvador, abandonado, sucio y con malas condiciones de suelo. Luego de esa ocupación del espacio, podemos advertir su territorialización: "es nuestro barrio, nosotros lo hicimos, nosotros tenemos que defenderlo y cuidarlo", "más de una vez nos han querido sacar, pero de ahí no nos mueve nadie, ese barrio lo hicimos nosotros",

\footnotetext{
${ }^{13}$ Las autoras Bergesio y Golovanevsky defienden el tratamiento de San Salvador de Jujuy como una ciudad intermedia por su peso en la población de la provincia (Bergesio; Golovanevsky, 2014:13).

${ }^{14}$ Es un sector ubicado en la zona sur de la ciudad de San Salvador de Jujuy, a 4 km de dicha ciudad, que alberga los barrios Sargento Cabral, Éxodo Jujeño y Balcón del Comedero, entre otros. Este sector concentra aproximadamente la tercera parte de la población total de San Salvador de Jujuy y tiene un alto crecimiento demográfico ya que permanentemente recibe migrantes del interior de la provincia de Jujuy, de la vecina provincia de Salta y de países con los que limita.
} 
o frases similares son habitualmente enunciadas por los entrevistados de la Tupac Amaru.

La organización pasa a controlar ese espacio, ahora transformado en territorio. Una buena parte del barrio Alto comedero, está conformado por el barrio de viviendas de la Tupac Amaru. Tiene allí construidas 3000 viviendas y le llaman (y así se lee en su entrada principal) el "cantri" de la Tupac $^{15}$, tiene muy claramente diferenciado su perímetro, sus casas son todas iguales, en el interior del barrio se dispone de una escuela y guardería infantil, un polideportivo y un parque acuático (con la pileta más grande de todo el noroeste argentino), un Centro cultural (que cuenta incluso con un cine), un centro de salud, el Centro Modelo Integral de Rehabilitación (CEMIR), una fábrica textil, una de bloques, una de muebles de caño, una metalúrgica y otros emprendimientos. Todos espacios territorializados: construidos y gestionados por la organización. Este mismo esquema es replicado por la organización en los diversos barrios de San Salvador y en las diversas localidades donde tiene desarrollo.

Esos territorios son también espacios institucionales: se crearon “pautas regularizadas de conducta e interacción conocidas, practicadas y aceptadas por los actores cuya expectativa es seguir actuando de acuerdo con dichas reglas" (O'Donnell, 1997: 310). Todos esos servicios y trabajos funcionan como espacios institucionales del barrio, los cuales desarrollan sus actividades específicas en materia de educación, salud, cultura, deportes, recreación.

Dichas instituciones crean un territorio que se solapa con el territorio de acción estatal. Se trata de servicios brindados por una organización

\footnotetext{
${ }^{15}$ Aludiendo mediante la castellanización del nombre en inglés de las urbanizaciones cerradas propias de sectores de altos recursos (country), a una similar disposición del espacio: claramente delimitado y conteniendo en su interior prácticamente todos los servicios que las familias que viven en él puedan necesitar, aunque, por supuesto, con una impronta claramente popular.
} 
que es parte de la "sociedad civil", servicios brindados en forma gratuita y que, de cierta manera, se ubican en un espacio de superposición con el Estado provincial: no compiten con él, puesto que la monopolización de la coerción no es un atributo que se halle directamente desafiado (ante un delito cometido en el barrio, por ejemplo, actúa la política y la justicia oficiales) aunque podríamos pensar en situaciones en las cuales indirectamente sí lo es, mediante las "sanciones" que efectivamente ordenan el funcionamiento institucional en este territorio controlado casi con exclusividad por la Tupac Amaru ${ }^{16}$. No se compite frontal y abiertamente con el Estado, pero estas acciones territorializadas ponen en suspenso muchas de sus atribuciones y genera innumerables tensiones.

Si analizamos la Tupac en tanto actor político, es posible sostener que, para quien pretenda gobernar la ciudad de Jujuy, resulta insoslayable decidir una forma de relacionamiento con la Organización Barrial Tupac Amaru, puesto que no sólo posee un poder de movilización que difícilmente pueda superar otra organización social o política de la provincia (incluyendo aquí a partidos, sindicatos y otras movimientos sociales) sino que posee un desarrollo territorial que puede hacer tambalear la legitimidad del Estado para actuar en ciertos territorios, delimitados y controlados en muchos aspectos directamente por la organización.

La impronta tupaquera conjuga de manera ciertamente particular diversos elementos en una identidad propia. En primer lugar, una fuerte raigambre popular, en ocasiones asociada también a la indianidad, aunque desde un punto de vista básicamente discursivo. Se trata de una organización nutrida por los sectores sociales más desprotegidos histórica-

\footnotetext{
${ }_{16}$ Algunas de estas sanciones pueden ejemplificarse: desde echar del Alto comedero a un traficante que vivía en el barrio, pasando por un llamado de atención frente a una asamblea a quien no ha cumplido con el horario de trabajo o no ha colaborado con la copa de leche de la cual forma parte, hasta ser "castigado" y bajar de categoría en el "escalafón laboral" de la organización: pasar de ser cooperativista a trabajar nuevamente en la copa de leche.
} 
mente en Jujuy, particularmente afectados por las políticas neoliberales. Sectores excluidos material y simbólicamente ${ }^{17}$ que son incorporados a una identidad política novedosa. En segundo lugar, una impronta eficientista y antiintelectual que posee cierto emparentamiento con la filosofía liberal: "no nos importan las definiciones, los debates intelectuales a ver si somos esto o aquello, que ideología tenemos...nos importa hacer las cosas, póngannos el nombre que quieran" (Ricardo, S. S. de Jujuy, 09/04/15). En tercer lugar, una estructura de liderazgo fuertemente personalista y jerárquico, que concentra en la figura de su líder no sólo casi todas las decisiones, sino también la fuente de la relación afectiva y de unidad, casi con una impronta maternal; combinada con una plataforma organizativa con anclaje territorial.

Propongo comprender la institucionalización de dicha identidad así construida a través de su dispositivo de poder territorial, es decir, es posible institucionalizar dicha identidad, manteniendo su potencia política transformadora, por su desarrollo territorial. Se trata de un colectivo que territorializa el espacio en el cual se asienta, porque produce su espacio: se apropia y domina el espacio, definiéndolo como un territorio propio, bajo su control, definiendo sus usos posibles y aceptados. El desarrollo, crecimiento y capacidad de movilización de la organización se asienta en dicho proceso de territorialización, su misma posibilidad de existencia y crecimiento se nutre de dicha lucha por el territorio, por lo que entiendo se lo puede considerar un movimiento socioterritorial (Fernandes, 2005).

Esta territorialización protagonizada por una organización social, habilita el análisis de la producción del espacio social, redefinido a partir de una propuesta y una identidad política que, para poder desarrollarse,

\footnotetext{
${ }_{17}$ Una de las reacciones de la Tupac ante dicha marginación simbólica se materializó en la construcción de piletas de natación en casi todas las localidades en las cuales tiene desarrollo. Es la respuesta a años de impedimento a los niños pobres de Jujuy de acceder a las piletas "publicas".
} 
construye un territorio. El espacio se redefine a partir de prácticas espaciales de resistencia que permiten la constitución de espacios de identificación transformadores que pueden institucionalizarse como nuevas formas de representación y acción política. ${ }^{18}$

\section{Palabras finales}

Decimos que algunos de los dispositivos institucionales que desarrollan ciertos movimientos sociales, como el caso de la OBTA brevemente reseñado, tienen un carácter territorial, porque es en la disputa y/o solapamiento territorial con el Estado (sea ésta resuelta luego bajo el signo de la colaboración y/o la confrontación) donde dichos dispositivos pueden sancionarse y aplicarse. Nos resulta muy sugerente la formulación de Pablo Stefanoni, para analizar el proceso de transformación en Bolivia:

Frente a las polarizadas visiones actuales acerca de cómo "cambiar el mundo" el MAS-IPSP plantea sin fisuras la necesidad de transformar el poder conquistado por las organizaciones sociales en poder estatal. En un "devenir Estado" cuyas bases materiales pueden visualizarse en las funciones paraestatales que corrientemente los sindicatos campesinos - y las instituciones tradicionales (ayllus, etc.) - cumplen en las áreas rurales bolivianas desde los años cincuenta (Stefanoni, 2008:.370-371).

\footnotetext{
${ }^{18} \mathrm{Al}$ momento de remitir el presente trabajo para su edición final por la revista, la Organización Barrial Tupac Amaru se encuentra atravesando un momento de grave tensión, conflicto y resistencia ante el gobierno provincial y nacional. La detención de su principal dirigente, Milagro Sala desde enero de 2016, a la que se sumó la de otros miembros referentes de la organización, es el dato más saliente y doloroso de un proceso integral protagonizado por los mencionados gobernantes, buscando aniquilar a la Tupac Amaru en todo el país y especialmente en la provincia de Jujuy. La fortaleza del movimiento y su capacidad de resistencia están siendo puestas en juego, bajo la posibilidad que una organización como la Tupac, nacida como respuesta a múltiples necesidades materiales y simbólicas de los sectores más humildes de una provincia postergada por décadas, pueda seguir articulando una alternativa política popular.
} 
Estas "otras" institucionalizaciones "devienen Estado" a pesar de no poseer sanción legal-republicana y no asentarse necesariamente en un fundamento universal, lo que las distancia de las instituciones propias de la construcción ciudadana... sin embargo, desde el particularismo que define la necesidad, estas organizaciones sociales territoriales funcionan "reemplazando", colaborando o compitiendo con el Estado en sus funciones sociales. Proponemos, entonces, comprender la noción de éstos procesos de institucionalización social a partir de un dispositivo territorial que se transforma en el único posible.

Coincidimos con Tapia, cuando habla de "la política del desborde" para describir el proceso mediante el cual, ante una demanda no cubierta por parte de los órganos institucionalizados de la política, fundamentalmente el Estado, se resuelve por otras vías: Las formas de vida política que no se organizan como parte del gobierno, se organizan para disputarle y controlar ese ejercicio del poder político (Tapia, 2008: 3). Sin embargo, nos distanciamos del análisis del autor boliviano cuando sostiene que

En tanto hay movilización de fuerzas, demandas y proyectos, se ocupan lugares, hay un recorrido de las acciones, pero éstas tienden a no estabilizarse e identificarse con un lugar delimitado e institucionalizado de la política; cuando ocurre esto, se vuelven simple sociedad civil. En este sentido, el campo de fuerzas configurado por los movimientos sociales es un no lugar político; es una zona de tránsito del conflicto social...

Como intentamos demostrar a lo largo de este trabajo, hay procesos de institucionalización de la política, aun cuando esta institucionalización sea considerada informal, que permiten pensar en un 'otro' lugar de la política y 'otra' institucionalización. Consideramos que esta otra institucionalización es posibilitada por procesos de territorialización y es válida para pensar algunas de las formas de intervención política que desarrollan 
los movimientos sociales, en un complejo entramado de interacciones con el Estado.

Fernanda Valeria Torres es Doctora en Ciencias Sociales, Profesora de grado y posgrado en la Facultad de Humanidades y Ciencias de la Educación (UNLP) e Investigadora del CONICET. $\bigotimes$ fernandav_torres@yahoo.com.ar

\section{Referencias}

1. ABERS, R.; VON BÜLOW, M. Movimentos sociais na teoria e na prática: como estudar o ativismo através da fronteira entre Estado e sociedade? Sociologias, Porto Alegre, ano 13, 2011, no 28: 52-84.

2. BERGESIO, L.; GOLOVANEVSKY, L. Las ciudades y sus muros de cristal. Ajuste neoliberal en una experiencia del noroeste argentino. Economía, Sociedad y Territorio, vol. xiv, núm. 44, 2014, 1-48.

3. CARLOS, E. Movimentos sociais: revisitando a participação e a institucionalização. Lua Nova, São Paulo, 2011, 84: 353-364.

4. CARLOS, E. Movimentos sociais e instituições participativas. Efeitos do engajamento institucional nos padrões de ação coletiva. RBCS Vol. 30, 2015, n 88: 83-99.

5. DOWBOR, M. Ocupando o Estado: análise da atuação do Movimento Sanitário nas décadas de 1970 e 1980. In: CARLOS, E.; PORTO DE OLIVEIRA, O.; ROMÂO, W. M. (orgs.) Sociedade Civil e Políticas Públicas. Atores e instituições no Brasil contemporâneo, Chapecó: Editorial Argos, 2014.

6. FERNANDES, B.M. Movimientos socio-territoriales y movimientos socio-espaciales. In: OSAL. Buenos Aires: CLACSO, 2005, n¹6: 273-283.

7. FERNANDES, B.M. Sobre a tipologia de territórios. In: SAQUET, M. A.; SAVÉRIO SPOSITO, E. (org.) Territórios e territorialidades: teorias, processos e conflitos. São Paulo: Expressão Popular, UNESP, 2009.

8. HAESBAERT, R. O mito da desterritorialização. Rio de Janeiro: Bertrand Brasil, 2004.

9. HIERNAUX, D.; LINDÓN, A. Desterritorialización y reterritorialización metropolitana: la ciudad de México. Documents d'Anàlisi Geogràfica, nº44: 71-88. Barcelona: UAB, 2004. 
10. LAGOS, M. (Dir.) Jujuy bajo el signo neoliberal. Política, sociedad y cultura en la década del noventa. Jujuy: Universidad Nacional de Jujuy, EdiUNJu, 2009.

11. LEFBVRE, H. The production of space. Oxford: Blackwell, 1991.

12. MACHADO ARÁOZ, H. Los dolores de Nuestra América y la condición neocolonial. Extractivismo y biopolítica de la expropiación. OSAL, Buenos Aires: CLACSO, N 32, 2012.

13. MCADAM, D.; TARROW, S.; TILLY, C. Dinámica de la contienda política, Barcelona: Hacer editorial, 2005.

14. MOSCOVICH, L. Fronteras entre la participación y la política. Estudio de las relaciones entre los gobiernos subnacionales y las organizaciones sociales con financiamiento federal en la Argentina. Presentado en XXI Congreso Internacional de Ciencia Política, Santiago de Chile, 2009.

15. O’DONNELL, G. Estado, democratización y ciudadanía. Nueva Sociedad, № 128, pp. 62-87, Caracas, 1993.

16. O`DONNELL, G. Otra institucionalización. In: O`DONNELL, G. Contrapuntos. Buenos Aires: Paidós, 1997.

17. OFFE, C. Reflexiones sobre la autotransformación institucional de la actividad política de los movimientos. Modelo provisional según estudios. In: DALTON, R. J.; KUECHLER, M. (comps.) Los nuevos movimientos sociales: un reto al orden político. Valencia: Edicions Alfons el Magnanim, 315-340, 1992.

18. ORGANIZACIÓN BARRIAL TUPAC AMARU (OBTA). Anuario "Nuestros 15 años". Jujuy: OBTA, 2014.

19. RAFFESTIN, C. Por uma geografía do poder. Sao Paulo: Editora Ática, 1993. 20. RONCONI, L. El programa Trabajar. Documento 63. Centro de Estudios para el Desarrollo Institucional - Fundación Gobierno y Sociedad, 2002. Consultado 4 de junio, 2015 http://faculty.udesa.edu.ar/tommasi/cedi/cedi.htm

21. SACK, R. Human Territoriality: Its Theory and History. Cambridge: Cambridge University Press, 1986.

22. SANTOS, M. La naturaleza del espacio. Barcelona: Ariel, 2000.

23. SILVA, M.; OLIVEIRA, G. A face oculta(da) dos movimentos sociais: trânsito institucional e intersecção Estado-Movimento - uma análise do movimento de Economia Solidária no Rio Grande do Sul. Sociologias, Porto Alegre, ano 13, 2011, no 28: 86-124. 
24. STEFANONI, P. El nacionalismo indígena como identidad política: la emergencia del MAS-IPSP (1995-2003). In: LEVY, B.; GIANATELLI, N. La política en movimiento. Identidades y experiencias de organización en América Latina. 311 378. Buenos Aires: CLACSO, 2008.

25. SVAMPA, M. Consenso de los commodities, giro ecoterritorial y pensamiento crítico en América Latina. OSAL, Buenos Aires: CLACSO, N 32, 2012, pp. 15-38. 26. TAPIA, L. Movimientos sociales, movimientos societales y los no lugares de la política. In: TAPIA, L. Política Salvaje, La Paz: CLACSO, Muela del Diablo Editores y Comuna, 2008.

27. TARROW, S. El poder en movimiento: Ios movimientos sociales, la acción colectiva y la política. Madrid: Alianza, 1997.

28. TILLY, C. Los movimientos sociales, 1768-2008. Desde sus orígenes a facebook. Barcelona: Crítica, 2010.

29. TOURAINE, A. An introduction to the study of social movements. In: Social Research, 52, 1985, p. 749-788.

Recebido: 18.06.2015

Aceite: 26.03.2016 